After much suppuration, their good effects depend in a great measure on the amount of the disease of the bone. If the whole shaft becomes necrosed, of course the sore will not heal till this has been got rid of ; but suppuration of ten occurs and yet but little, or perhaps none, of the bone dies. In such a case the sulphides hasten the expulsion of the pus, and if the skin is already broken before they are employed, they improve the character of the wound and the discharge, and the sore heals, leaving a sunken scar adherent to the bone, whilst the finger slowly assumes its natural proportions. Large indolent abscesses may form on the back of the hands or feet. These are similarly affected by the sulphides. Whilst these remedies are thus influencing locally these strumous formations and abscesses, the child's health greatly improves, although failing previously, in spite, perhaps, of the administration of cod-liver oil and steel wine. That the improvement is due to the sulphide is shown by the fact that the amendment occurs where only this drug is administered. On prematurely discontinuing the sulphide, fresh formations are apt to appear especially on the occurrence even of a slight, illness; indeed, a severe illness will often excite a few fresh abscesses, in spite of the sulphides.

The sulphides appear to me to exercise a very beneficial influence in suppurating scrofulous glands in the neck. Here again they hasten the elimination of the pus, and subsequently the cheesy scrofulous matter. After the abscesses have burst, and continued slowly discharging a scanty, unhealthy pus, and when the edges of the sores have become much thickened and indurated, these remedies render the discharge more abundant, thick, creamy, and healthy, considerably hasten the evacuation of the scrofulous matter, which prevents the healing of the wound, and at the same time softens the round indurated edges, so that the sore heals much more speedily. If small doses appear to affect these sores but little, larger doses, as half a grain or a grain, should be given several times a day, or even every two hours. I need hardly say that to compass the results described the treatment must be continued several weeks, for it is vain to expect them to occur in a few days, when the sores have been discharging perhaps for months or even years.

The topical effect of sulphur ointment, or of an ointment of the hypochlorite of sulphur, or, still better, of the iodide of sulphur of the Pharmacopoeia, is most marked on acne indurata and acne rosacea. Here, again, the effects are twofold, and even opposite, according to the stage of the eruption. If applied at the very commencement of the eruption, as soon as the little hard knot is felt under the skin, further development is arrested, and the hardness speedily disappears. For instance, if smeared over the hardness just before going to bed, in the morning scarcely any induration will be felt, though after a time, perhaps from exercise, or the irritation from washing, much of the hardness may return, to be again removed by a renewed application of the ointment, so that in two or at most three days a papule that threatened to become of considerable size may be completely dispersed. When, however, the nodule has advanced further, and suppuration has set in, then the effects of the ointment are much like those of sulphides, administered internally, on boils. The ointment hastens maturation, limits the swelling and hardness, and thus considerably curtails the duration of the eruption. Nay, further, if rubbed over the skin it appears to check the formation of the acne spots. If rubbed over the nose and neighbouring parts of the face in acne rosacea its effects are often striking. Not only does it act as in acne indurata, but the hardened, swollen tissues become softened and reduced to a more natural state. I have found the iodide of sulphur likewise useful in bromic acne, reducing the eruption, or at least considerably lessening the size of each spot. In acne the ointment should be thickly smeared over the eruption night and morning.

Anyone who gives the sulphides a fair trial in the foregoing cases will, I feel confident, have reason to be gratified with the result.

Carendish-place, W.

At a late meeting of the Guardians of the Enniscorthy Union, Dr. O'Rourke, late medical officer of the workhouse, was granted a retiring allowance equivalent to two-thirds of his salary.

\section{A DESCRIPTION OF A SIMPLE APPARATUS FOR MAINTAINING A FLEXIBLE CATHETER IN POSITION.}

\section{Br J. C. OGILVIE WILL, M.D.}

IN treating urethral stricture by the process generally, though erroneously, termed gradual mechanical dilatationfor which mode of treatment I entertain a special liking, as the results I have myself obtained from its use, and those which I have had opportunities of observing in the practice of others, have convinced me that in most cases a safer and more efficient method could not be desired-I have considered it expedient, in some very narrow, tight strictures, to secure a gum-elastic catheter in the urethra for a period of twentyfour or forty-eight hours, when I have invariably found that absorption of the substance forming the stricture has been induced to such an extent as to enable an instrument several sizes larger to be passed with facility.

The methods generally used for the purpose of securing such an instrument in position-viz, threads of worsted, tapes, \&c.-seemed to me to be clumsy and dirty ; I therefore devised the little appliance of which $I$ append a drawing, and I consider that it possesses the recommendations of neatness, cleanliness, and efficiency. It consists, as may be seen from the woodcut, of a collar or ring, with two slips attached to it. When employing it, the prepuce having

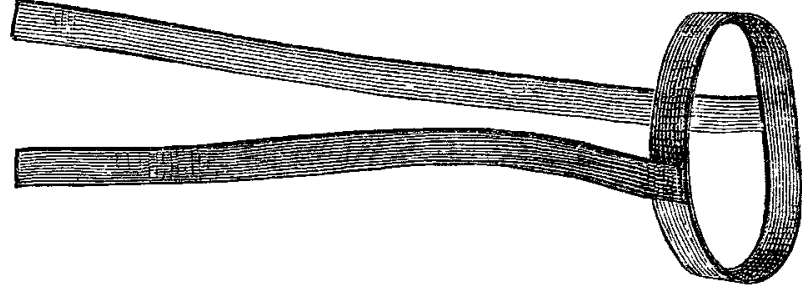

been retracted, the ring should be put slightly on the stretch, and passed over the glans penis into the constriction or cervix behind the corona; the slips are then to be brought forward and attached to the calheter by a thread or by means of a little gutta-percha solution.

The apparatus, as depicted, appears more formidable than it really is, and it is probably larger, and the slips far longer, than would be required; the woodcut may therefore be regarded as an altogether magnified representation.

The substance from which the ring is made is a thin, soft, elastic material, generally known as dentists' rubber sheet; the slips of the same material, or should their elasticity be objected to, which it certainly would be in some cases in which the retention of a flexible catheter is a desideratum, common rubber sheet, which can be had extremely fine and soft, yet unyielding, may be substituted.

The construction of the appliance of the size necessary is the work of a few moments, all that has to be done being the cutting of narrow strips from a sheet of the material mentioned, and fixing them in their relative position by smearing the ends with a little gutta-percha solution and pressing them together. The rings of rulcanised indiarubber generally met with are not nearly so suitable as those I have described, as from the manner in which they are shaped they cannot be applied with such a degree of accuracy as to ensure that they will neither, on the one hand, constrict too tightly, nor, on the other, lose their hold and slip forward. Though I have only indicated the use of the elastic collar in maintaining a catheter in situ during the treatment of stricture, it is of course equally applicable to many other cases in which the presence of a flexible instrument in the bladder is deemed advisable.

In connexion with this subject, let me call to remembrance the fact that in all cases in which the retention of a flexible catheter is required, the object will be much more surely attained if the plug be so fashioned that it can be passed into the instrument for a distance of at least an inch; it will thus be made to answer the double purpose of maintaining the catheter in position and preventing the egress of urine.

The materials used in preparing the apparatus may be obtained from Messrs. Young and Son, cutlers, North Bridge, Edinburgh.

Union-terrace, Aberdeen. 